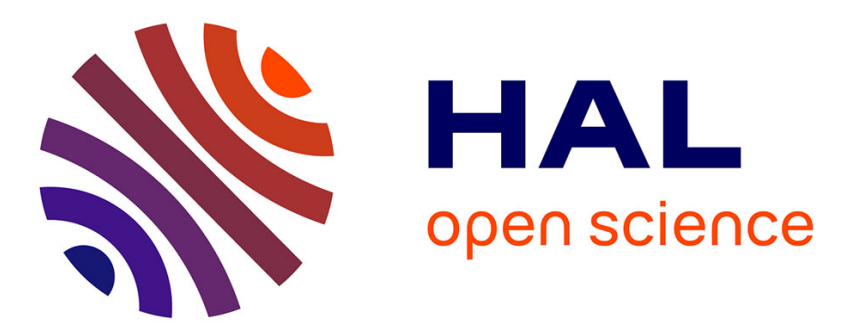

\title{
Comparison of models to analyze mortality data and derive concentration-time response relationship of inhaled chemicals
}

Alexandre R.R. Pery, Adrien Troise, Sylvie Tissot, Jean-Marc Vincent

\section{To cite this version:}

Alexandre R.R. Pery, Adrien Troise, Sylvie Tissot, Jean-Marc Vincent. Comparison of models to analyze mortality data and derive concentration-time response relationship of inhaled chemicals. Regulatory Toxicology and Pharmacology, 2010, 57 (1), pp.124-128. 10.1016/j.yrtph.2010.02.005 . ineris00961747

HAL Id: ineris-00961747

https://hal-ineris.archives-ouvertes.fr/ineris-00961747

Submitted on 20 Mar 2014

HAL is a multi-disciplinary open access archive for the deposit and dissemination of scientific research documents, whether they are published or not. The documents may come from teaching and research institutions in France or abroad, or from public or private research centers.
L'archive ouverte pluridisciplinaire HAL, est destinée au dépôt et à la diffusion de documents scientifiques de niveau recherche, publiés ou non, émanant des établissements d'enseignement et de recherche français ou étrangers, des laboratoires publics ou privés. 


\title{
Comparison of models to analyze mortality data and derive
}

\section{concentration-time response relationship of inhaled chemicals}

\author{
Alexandre R.R Péry ${ }^{a^{*}}$, A. Troise ${ }^{a, b}$, S. Tissot ${ }^{b}$ and J.M. Vincent ${ }^{b}$
}

INERIS BP2, Parc Technologique Alata 60550 Verneuil-en-Halatte, France

${ }^{a}$ Unité Modèles pour l'Ecotoxicologie et la Toxicologie (METO),

${ }^{\mathrm{b}}$ Unité d'Expertise et Evaluations en Toxicologie (ETSC)

${ }^{*}$ Corresponding author. Address: INERIS, BP2, Parc Alata 60550 Verneuil-en-Halatte,

France. Tel. +33-3-44-61 8144 Fax: +33-3-44-55-68-00. E-mail address:

alexandre.pery@ineris.fr 


\begin{abstract}
The derivation of thresholds for lethal effects for inhaled chemicals is a key issue in accidental risk management because they largely determine the outcome of land use planning, among which localization of habitations in the vicinity of a factory. This derivation is generally performed on the basis of rodent lethality data analyzed by statistical models able to extrapolate effects for different times and concentrations of exposure. A model commonly used in France is the standard probit model. In this model, effects is related to exposure concentration and duration according to the Haber's law and considers that individual thresholds, corresponding to the maximum tolerated effects before dying, are log-normally distributed among the population. This approach has been criticized for its lack of biological parameters and its inability to treat data characterized by only one time of exposure. In order to improve the current state of modeling, we proposed three alternative models. Two of them (DEBtox and Haber TKTD models) incorporate the kinetics of the chemicals. The third one (Loguniform model) is a linearization of the standard probit model. We evaluated their performance by analyzing real data and simulated data generated with each model. For data characterized by several times of exposure, the standard probit model outperformed all other models in terms of goodness of fits and estimation of parameters. For data characterized by only one time of exposure, only DEBtox model was able to fit the data and estimate parameters, provided we dispose of several observation times, typically just after exposure and a long period afterwards.
\end{abstract}

Keywords: accidental risk; modeling; lethality data; parameters estimation; probit. 


\section{Introduction}

To prevent risks related to accidental releases of dangerous chemical substances in the atmosphere, risk managers need acute toxicity thresholds in association with accident scenarios to produce safety reports and design emergency plans. In France, they usually determine the zones of lethal, irreversible and reversible effects relative to the location of plants storing, producing or using toxic substances, especially for land use planning. The lethality threshold is related to a certain percentage of death occurring during the experimental test or in the following fourteen days post exposure (including animal sacrificed for ethical reasons). The "irreversible effects" correspond to the persistence over time of a lesion or a functional damage induced by an exposure. Three types of irreversible effects are pointed out, lesion without functional repercussions, lesion with functional repercussions (like bronchopneumopathy, pulmonary fibrosis, necrosis of olfactory epithelium with anosmia) and the functional irreversible impairment (like asthma). The "reversible effects" correspond to a return to the level of health prior to exposure (immediately or in a reasonable time after). It is therefore crucial to evaluate properly the thresholds, because they determine the distances of effects. Indeed, if the thresholds are overestimated, distances are overprotective with economic impact. In contrast, if the thresholds are underestimated, the health of the exposed population is threatened.

A French methodology was developed to evaluate the quality of the data available and to deduce from these data acute toxicity thresholds. It comprises many steps. The first step is the selection of an experimental key study (mainly, an animal study) for each effect, based on the method developed by Klimisch et al. (1997) for quality assessment and on expert judgment for relevance of the observed effects related to the type of threshold. The second step consists in finding the relevant critical toxic effect for the two types of effects which are considered in addition to lethal effects, i.e. irreversible and reversible effects. The third step is the data 
analysis based on a statistical model. The fourth step considers the extrapolation from animals to humans (with or without uncertainty factors). Here we focus on lethality data only. Usually, the data to analyze are rodent lethality measurements for different exposure concentrations and different exposure durations, observed after at least a fourteen-day period. It can however happen that the information is available only for single exposure duration. Standard dose-response models are generally based on the Haber's law, or its generalizations (ten Berge et al., 1986). These generalizations state that the effect for exposure to concentration $C$ during a period of time $t$ is a function of $\left(C^{n} t\right)$, named fixed effect level (Jarabek, 1995), where $n$ is called the Haber constant. Thus, in the standard probit model, the probability of death equals:

$$
P(\text { death })=F\left(\frac{n \log (C)+\log (t)-\mu)}{\sigma}\right)
$$

where $F$ is the cumulative distribution function of a normal distribution with mean $\mu$ and standard deviation $\sigma$ (Diack and Bois, 2005). In this formulation, each individual has a given threshold, log-normally distributed. If he is exposed to an effect level exceeding this threshold, death will occur.

There are many limits to this model. First, it is not possible to analyze data for which only one exposure time is available, because only two combinations of parameters $(n / \sigma$ and $(\log (t)-$ $\mu) / \sigma$ ) could be identified in this case. This model has also been criticized for its lack of biological parameters by Diack and Bois (2005) who have proposed an alternative model (the so-called PKPD model). However, the model they propose has even more limitations in a risk assessment perspective.

First, the gain in terms of realism compared to the standard probit approach is far from obvious. Indeed, the proposed equation for kinetics is the following one:

$$
\frac{d Q}{d t}=k a C^{n}-k e Q
$$


with $Q$ the internal quantity of substance in the tissue, $C$ the exposure concentration, $k_{a}$ the pulmonary ventilation rate, $k_{e}$ the elimination rate, and $n$ an unknown parameter analogous to the Haber constant. This is a very unusual kinetics equation, because there is no reason for the parameter $n$ to be different from 1, i.e. for the intake rate of the substance not to be proportional to its concentration in the air. Authors try to justify their equation with some biological consideration in their discussion, but their reasoning would be acceptable only for very rapid kinetics.

Second, there are five parameters to estimate $(k a, k e, n$, but also $\mu$ and $\sigma$ as in the probit model), compared to the three ones in the probit approach. In practice, when analyzing data for accidental risk assessment, with both the PKPD model and the standard probit model, we found that the common parameters have similar estimates but that the remaining parameters have large confidence intervals. This observation indicates an excessive number of parameters to estimate. In particular, the estimation of the kinetics parameters is not feasible when only data for one time of exposure are available.

Here, we propose to evaluate three alternative models. These models have the same numbers of parameters (three) as the standard probit model. The first two models incorporate chemical kinetics through a one-compartment model to add realism relative to compound uptake and elimination. They differ in the toxicodynamics part. The first one, which is called DEBtox (Kooijman and Bedaux, 1994), is based on a threshold approach which has already been used in ecotoxicology. The second one is based on the Haber's law and will be here referred to as Haber TKTD. The third approach we propose is an approximation of the probit standard by using a loguniform statistical distribution for $\mathrm{F}$ instead of a normal one. The comparison of the models we propose is based on the analysis of datasets generated from simulations with each of the models and on actual data which have already been used to derive toxicity threshold for accidental risk assessment. 


\section{Materials and Methods}

\subsection{Mathematicals models to analyse survival data}

\subsubsection{Standard probit}

As already presented in the introduction, in the standard probit model, the probability of death equals:

$P($ death $)=F\left(\frac{n \log (C)+\log (t)-\mu}{\sigma}\right)$

where $F$ is the cumulative distribution function of a normal distribution with mean $\mu$ and standard deviation $\sigma$. It consequently assumes that the individual threshold for response follows a lognormal distribution. When the product $C^{n} t$ exceeds its threshold, the individual dies.

\subsubsection{Loguniform model}

In this model, the probability of death equals:

$$
P(\text { death })=F\left(\frac{n \log (C)+\log (t)-a}{b-a}\right)
$$

where $F$ is the cumulative distribution function of an uniform distribution with lower and upper bounds 0 and 1, and where $a$ and $b$ are bounds of the response. As for the standard probit, when the product $C^{n} t$ exceeds the threshold of an individual, the individual dies. There is no individual having a threshold below $a$ and above $b$, which means that no individual are expected to die for $C^{n} t$ values below $a$ and no individual are expected to survive for $C^{n} t$ values over $b$. The Loguniform model can be seen as a linearization of the standard probit model. The comparison will permit to assess the relevance of the choice of the statistical distribution for $F$.

\subsubsection{DEBtox model}


DEBtox model is a mathematical model that has first been developed to analyse aquatic ecotoxicological survival data. It has been proposed by Kooijman and Bedaux (1996). It is particularly adapted for the analysis of toxicological data obtained under time varying exposures (Pery et al., 2001; Pery et al., 2002).

In DEBtox model, a kinetics module, accounting for the dynamics of compound body concentration is coupled with an effects module. To keep the same number of parameters as for the standard probit, we assumed, as in the standard DEBtox model, that all individuals have a common threshold for effects. Once this threshold is exceeded by the dose at target organ level, the probability to die is not $100 \%$ but increases linearly as a function of this dose. Dose at target level is described using the following linear one-compartment kinetics model:

$$
\frac{d c_{i}}{d t}(t)=k_{e}\left(C(t)-c_{i}(t)\right)
$$

where $k_{\mathrm{e}}$ is the elimination rate, $C$ is the concentration measured in exposure air, and $c_{\mathrm{i}}$ the scaled body concentration. This parameter corresponds to the ratio of the amount of compound in the whole body to the body volume. It is scaled by the bioconcentration factor (a constant corresponding to the ratio of the concentration in the target organ to the concentration in air at toxicokinetics steady state) in order to ensure the feasibility of parameters estimation. Toxic effects occur only when $c_{\mathrm{i}}(t)$ exceeds a threshold, the $N E C$ (No Effect Concentration), which corresponds to the maximal toxicant concentration at target organ level that can be handled by regulation systems without generating detectable effects on mortality. Survival probability in exposed organisms is described based on $c_{\mathrm{i}}(t)$, which drives toxicodynamics. Death being assumed to be a stochastic process, the probability $q(t)$ to survive until time $t$ is defined as:

$$
q(t)=\exp \left(-\int_{0}^{t} h(\tau) d \tau\right)
$$


where $h(\tau)$ is the hazard rate at time $\tau$. For a small time interval $\mathrm{d} \tau, h(\tau) \mathrm{d} \tau$ represents the probability of dying between $\tau$ and $\tau+\mathrm{d} \tau$, for an organism which has survived until time $\tau$. Toxicant hazard linearly increases with the difference between $c_{\mathrm{i}}(t)$ and the threshold value when this threshold is exceeded:

$\left\{\begin{array}{l}\text { if }\left(c_{i}(t)>N E C\right), h(t)=b\left(c_{i}(t)-N E C\right) \\ \text { if }\left(c_{i}(t)<N E C\right), h(t)=0\end{array}\right.$

where $b$ is the killing rate, a descriptor of the intensity of effects. The effects data permit both to estimate the parameters $b$ and $N E C$, but also the kinetics parameter. For instance, under constant exposure, it is related to the time at which the level of effects reaches a constant value.

\subsubsection{Haber-TKTD model}

This model has the same kinetics module as the DEBtox model. It differs from the latter model in the effects module, with the following definition for $h$ :

$$
h(t)=b C(t)^{n}
$$

where $b$ is a proportionality constant, and $n$ is the Haber constant.

It is noteworthy to realize that, in case of $n=1$, we have, for high values of $t$, corresponding for instance to observation times (typically 14 days, whereas exposure is limited to 8 hours maximum):

$q(t)=\exp (-b C t)$

whatever the value for $k_{e}$. This means that, in this case, effects are related to the Haber's law, regardless of the actual compound kinetics.

\subsection{Data analyzed in this study}

\subsubsection{Simulated data}

To test the ability of the models for data analysis, we simulated datasets according to each model and analyzed these data with all the models. We aimed at producing realistic data, with 
small effects for low exposure times and low exposure concentrations and high effects for high exposure times and high exposure concentrations. We used four concentrations $(50,100$, 200 and $300 \mathrm{ppm})$ and four times of exposure (30,60, 120 and 240 minutes). We generated twenty different data sets per model. For each condition in each dataset, we simulated the results for 10 individuals. First, we calculated the mean probability for an individual to be dead at the observation time. Using a random generator (from the commercial software Excel), we simulated mortality data by comparing, for each individual, the number generated (between 0 and 1) and the probability to be dead. If the random number was below this probably, the outcome for this individual was death.

For better comparison of performance of the different models, we chose parameters so that the theoretical value for the $5 \%$ letal concentration $\left(5 \% \mathrm{LC}_{30 \mathrm{~min}}\right.$, which is the concentration for which the mean expectation is $5 \%$ mortality after 30 minutes of exposure) is $100 \mathrm{ppm}$ for all the simulations. The $5 \% \mathrm{LC}_{30}$ min is of great interest in accidental risk assessment.

For each model, two sets of parameters values were used. The parameters values we selected for the simulations were $n=3, \mu=7.43, \sigma=1$, and $n=1, \mu=3.43, \sigma=1$, for the simulations based on standard probit model. This permits to cover the two options generally applied when fixing arbitrarily a value for $n$ to extrapolate between doses (ten Berge et al., 1986). With the same rationale, we chose $n=3, a=5.38, b=7.38$, and $n=1, a=1.38, b=3.38$ for the Loguniform model. For DEBtox model, we chose $k_{\mathrm{e}}=0.01 \mathrm{~min}^{-1}, b=0.0003 \mathrm{~min}^{-1} \mathrm{ppm}^{-1}$, $N E C=25 \mathrm{ppm}$, and $k_{\mathrm{e}}=10 \mathrm{~min}^{-1}, b=0.0000228 \mathrm{~min}^{-1} \mathrm{ppm}^{-1}, N E C=25 \mathrm{ppm}$, to have a simulation with slow kinetics and one with rapid kinetics. With the same rationale, we chose $k_{\mathrm{e}}=0.01 \mathrm{~min}^{-1}, b=0.0000022 \mathrm{~min}^{-1} \mathrm{ppm}^{-1}, n=2$, and $k_{\mathrm{e}}=10 \mathrm{~min}^{-1}, b=0.000000304 \mathrm{~min}^{-}$ ${ }^{1} \mathrm{ppm}^{-1}, n=2$, for the Haber-TKTD model.

We then evaluated if the models were able to fit correctly the data, to estimate the parameters, and to estimate properly the $5 \% \mathrm{LC}_{30 \mathrm{~min}}$. 


\subsubsection{Real data}

We analyzed data obtained for rats exposed to chemicals, with many times of exposure available to be able to use all our models, including those with kinetics. We had data for arsine (3 times of exposure, 5 to 7 concentrations, 10 female rats per condition) (IRDC, 1985a; IRDC, 1985b; IRDC, 1985c), cyanhydric acid (1 to 5 times of exposure, 4 to 39 concentrations, 10 rats per condition) (Ballantyne, 1994; Blagden, 1994; Higgins et al., 1972), sulfuric acid (4 times of exposure, 3 or 4 concentrations, 10 to 14 mice per condition) (Runckle and Hahn, 1976), hydrogen sulfide (1 to 4 times of exposure, 3 to 7 concentrations, 10 mice per condition) (Vernot and Mac Ewan, 1976; Zwart and Klokman, 1990), nitrogen dioxide (10 times of exposure, 7 concentrations, 5 to 14 mice per condition) (Hine et al., 1970), vinyl chloride ( 2 to 4 times of exposure, 3 to 14 concentrations, 5 to 90 mice per condition) (Mastromatteo et al., 1960; Prodan et al., 1975) and sulphur dioxide (3 to 4 times of exposure, 3 concentrations, 14 to 70 mice per condition) (Bitron and Aharonson, 1978). These data have already been used, with the standard probit approach, to derive thresholds used in accidental risk management.

\subsection{Estimation of parameters}

Parameters were estimated by maximizing the logarithm of the likelihood of the data, $L=\sum_{j=1}^{k}\left(n_{j} \ln \left(p_{j}\right)+\left(m_{j}-n_{j}\right) \ln \left(1-p_{j}\right)\right)$ where $p_{j}$ is the mean probability to be dead at the time of observation (14 days) for concentration $c_{j}$, calculated with the model selected for data analysis, $k$ the number of tested concentrations, $n_{j}$ the number of organisms dead at the time of observation for concentration $c_{j}, m_{j}$ the number of exposed organisms for concentration $c_{j}$, . To perform that maximization, we coded a program in $\mathrm{C}++$, which calculates the likelihood for combinations of large ranges of parameters, and selects the parameters providing the highest value. It is not time-saving but very robust, so that we avoid the selection of local maximums. 


\section{Results}

\subsection{Simulated data}

Table 1 presents the results of the analysis of the simulated data. Except in two cases (data simulated with Loguniform model $n=1$ and DEBtox with rapid kinetics), regardless of the model we used to simulate data, the standard probit model provides the best fit of the data. Moreover, the estimate of $n$ is never significantly different from the theoretical value (Wilcoxon test, $p<0.05$ ). Regarding the estimate of $5 \% \mathrm{LC}_{30 \mathrm{~min}}$, it is significantly different from the theoretical value only for one simulation (DEBtox and slow kinetics).

DEBtox and Haber-TKTD models provide correct fits and parameters estimation only when they have generated the data they analyze. It is important to note that, for data generated by DEBtox in case of rapid kinetics, it outperforms all other models. For the other simulations, fits are of very bad quality (with more than twice the sum of the squares of the deviations (SSD) of standard probit model).

Despite a relative good fit of the data (low SSD values), the Loguniform model provides biased estimates (significant differences between theoretical value and estimated values, for the $5 \% \mathrm{LC}_{30 \text { min }}$ in 6 of the 8 simulations), even when data have been generated with this model (case $n=3$ ).

Other simulations with different $\sigma$ and $a$ values (data not presented here) did not modify our conclusions.

\subsection{Real data}

Table 2 and 3 present the results of the analysis of the real data. To permit to compare the performance of fits between experiments, the SSD has been normalized by the number of animals used in the study. Except for cyanhydric acid, the fit is better using the standard probit model than the other ones. In contrast, Haber-TKTD model always provide the worst 
fits. The second best model is DEBtox in 5 cases over 7 ones and the Loguniform model in 2 cases over 7 ones. These results are in accordance with the analysis of simulated data. The value of $n$ estimated with the Loguniform model is close to the one estimated with the standard probit model except for sulfuric acid. From this point of view, the Loguniform model performs better than our expectations based on the study with simulated data.

\subsection{Possible use of the models in case of one exposure time only available}

As we showed in the introduction, it is not possible to estimate the parameters of the standard probit model for data characterized by only one time of exposure. This is also the case for the Loguniform model. Even setting the lower bound at a fixed value (corresponding, for instance, to an available NOAEL) would not permit to estimate the two remaining parameters. In contrast, for models with kinetics module, like DEBtox or Haber-TKTD, the parameters could be estimated in case of only one time of exposure, provided there are several observation times. We performed two additional datasets simulations, for each model, with 6 exposure concentrations (50,100, 150, 200, 250 and 300 ppm), only one time of exposure (60 min) but two observation times (60 min and 15 days). We used the same parameters as for the previous simulations in case of slow kinetics and produced 20 datasets per model. The estimation of the parameters of the Haber-TKTD model was not satisfactory with a huge variability. In contrast, DEBtox parameters were adequately estimated. The NEC estimate was $25 \pm 7 \mathrm{ppm}$, and the $5 \% \mathrm{LC}_{30}$ min estimate was $99 \pm 8 \mathrm{ppm}$ (the theoretical value is 100 $\mathrm{ppm}$ ), which is a better estimate than the one obtained for only one time of observation but several times of exposure (Table 1).

\section{Discussion}

When the data for many times of exposure are available, the standard probit model performed at best relative to goodness of fit. The description was the closest for real and simulated data, 
even, in the latter case, when the data have been simulated with other models. In contrast, the Haber-TKTD model was the worst of all the models we tested. DEBtox model was also not able to provide satisfactory fits in most simulated cases but was often the second best model when analyzing real data. The Loguniform model provided fits with a quality close to the one of the standard probit model, but it suffered from biases in the estimation.

For the analysis of the data usually used in an accidental risk assessment context, i.e. data with only one observation time but several times of exposure, we can conclude that better fits are obtained when accounting for variability of response between individuals than for kinetics during exposure and post-exposure period. This is coherent with our remark that the mathematical description of effects with the Haber-TKTD model does not depend on $k_{e}$ when $n$ equals 1 (See 2.1.4), as a consequence of measuring the data only when there is no more compound in the body. Models like DEBtox perform well when there are many measurements for different times of exposure, even if exposure concentrations are time-varying (Pery et al., 2001; Pery et al., 2002). For rodent lethality, measuring effects just after exposure and much later may be enough to improve the estimation of the kinetics parameter. Unfortunately, such data are seldom available. In the data we have, only those relative to Sulfur dioxide comprise several times of observation. We analyzed these data with DEBtox model. Sulfur dioxide was the chemical for which DEBtox performed at worst with data characterized by only one time of observation. However, with several observations throughout time, the sum of the squares of the deviations between mortality predictions and actual data normalized by dividing by the total number of exposed organisms in the study was divided by 1.8 compared to Table 2 . Moreover, the estimate for the $5 \% \mathrm{LC}_{30}$ min was $986 \mathrm{ppm}$, which is very similar to the estimate value using the standard probit model (1025 ppm).

The standard probit model is not able to analyze data for which only one time of exposure is available. In this case, the methodology used in France recommends the use of the probit 
standard to derive dose response for the only time of exposure available and the extrapolation of the results using Haber's law with $n=1$ for longer duration than the one available, and $n=3$ for shorter duration (ten Berge et al., 1986).

Among the models we studied, only DEBtox model would be able to analyze data characterized by only one time of exposure, provided mortality is reported at least two times, typically just after and long after exposure. However, it will be necessary to perform comparisons between DEBtox and standard probit models with real data and at least two observation times and several exposure times. This will permit to assess if the thresholds needed for accidental risk assessment are estimated with the same accuracy when applying DEBtox to data with several times of observation or applying the standard probit model to data with several times of exposure. Unfortunately, we could not find such data.

To conclude, it is recommended to use the standard probit model to analyze lethality data for accidental risk assessment, when data are available for many times of exposure. In contrast, there is no model that could replace the extrapolation with $n=1$ and $n=3$ to analyze data for only one time of exposure and extrapolate to other times if only one time of observation is available.

\section{Acknowledgements}

Authors would like to thank Enrico Mombelli, Céline Brochot and two anonymous reviewers for their comments on the study. This work was supported by the French Ministry in charge of Ecology and Sustainable Development for this study, within the framework of Programme 181.

\section{Conflicts of interest}

The authors declare that there are no conflicts of interest. 


\section{References}

Ballantyne, B., 1994. Acute inhalation toxicity of hydrogen cyanide vapor to the rat and rabbit. Toxic. Subst. J. 13, 263-282.

Bedaux, J.J.M., Kooijman, S.A.L.M., 1994. Statistical analysis of bioassays based on hazard modelling. Environ. Ecol. Stat. 1, 303-314.

Bitron, M.D., Aharonson, E.F., 1978. Delayed mortality of mice following inhalation of acute doses of $\mathrm{CH} 2 \mathrm{O}, \mathrm{SO} 2 \mathrm{Cl} 2$, and Br2. Am. Ind. Hyg. Assoc. J. 39, 129-38.

Blagden S.M., 1994. Hydrogen cyanide: multiple exposure time acute inhalation toxicity study in the rat. Rhone Poulenc-Secteur Agro, Sophia Antipolis, pp. 1-56.

Diack, C., Bois F.Y., 2005. Pharmacokinetic-pharmacodynamic models for categorical toxicity data. Regul. Toxicol. Pharmacol. 41, 55-65.

Higgins, E. A., Fiorca, V., Thomas, A.A., Davis, H.V. 1972., Acute toxicity of brief exposures to $\mathrm{HF}, \mathrm{HCl}, \mathrm{NO} 2$ and $\mathrm{HCN}$ with and without CO. Fire Technol. 8, 120-130. Hine, C. H., Meyers, F.H., Wright, R.W., 1970. Pulmonary changes in animals exposed to nitrogen dioxide, effects of acute exposures. Toxicol. Appl. Pharmacol. 16, 201-13. IRDC., 1985a. Arsine - LC50 acute inhalation toxicity evaluation in rats (30 min). International Research and Development Corporation, AT\&T Bell laboratories. IRDC., 1985b. Arsine - LC50 acute inhalation toxicity evaluation in rats (60 min). International Research and Development Corporation, AT\&T Bell laboratories. IRDC., 1985c. Arsine - LC50 acute inhalation toxicity evaluation in rats (240 min). International Research and Development Corporation, AT\&T Bell laboratories. Jarabek, A.M., 1995. Considerations of temporal toxicity challenges current default assumptions. Inhal. Toxicol. 7, 927-946. 
Klimisch, H.J., Andreae, E., Tillmann, U., 1997. A systematic approach for evaluating the quality of experimental toxicological and ecotoxicological data. Regul. Toxicol. Pharmacol. $25,1-5$.

Mastromatteo, E., Fisher, A.M., Christee, H., Danziger, H., 1960. Acute inhalation toxicity of vinyl chloride to laboratory animals. Am. Ind. Hyg. Assoc. J. 21, 394-8.

Péry, A.R.R., Bedaux, J.J.M., Zonneveld, C., Kooijman, S.A.L.M., 2001. Analysis of bioassays with time-varying concentrations, Wat. Res. 35, 3825-3832.

Péry, A.R.R., Flammarion, P., Vollat, B., Bedaux, J.J.M., Kooijman, S.A.L.M., Garric, J., 2002. Using a biology-based model (DEBtox) to analyse bioassays in ecotoxicology: opportunities and recommendations. Environ. Toxicol. Chem. 21, 459-465.

Prodan, L., Suciu, I., Pîslaru, V., Ilea, E., Pascu, L., 1975. Experimental acute toxicity of vinyl chloride (monochloroethene). Ann. N. Y. Acad. Sci. 246, 154-8.

Runckle, B. K., Hahn, F. F., 1976. The toxicity of H2SO4 aerosols to CD-1 mice and Fisher344 rats. Ann. Rep. Inhal. Toxicol. Res. Inst. 435-439.

ten Berge, W. F., Zwart, A., Appelman, L. M., 1986. Concentration--time mortality response relationship of irritant and systemically acting vapours and gases. J. Hazard. Mater. 13, 301309.

Vernot, E. H., Mac Ewan, J. D., 1976. Toxic hazards research unit annual technical report. AMRL report, Gaithersburg, MD, USA.

Zwart, A., Klokman, J. H. E., 1990. Determination of concentration time mortality relationships to replace LC50 values. Inhal. Toxicol. 2, 105-117. 
Table 1 Mean and standard deviations for the estimates of $5 \% \mathrm{LC}_{30}$ min (in ppm) and sum over all the simulated tests of the squares of the

deviation (SSD) between mortality predictions and actual data. The different columns account for the different models used for the simulations, and the different lines for the different models used to analyze the simulated data. For the models in which the Haber constant is involved, the

estimates for $n$ are presented.

\begin{tabular}{|c|c|c|c|c|c|c|c|c|c|c|c|c|}
\hline & \multicolumn{3}{|c|}{ Standard Probit $n=3$} & \multicolumn{3}{|c|}{ Standard Probit $n=1$} & \multicolumn{3}{|c|}{ Loguniform $n=3$} & \multicolumn{3}{|c|}{ Loguniform $n=1$} \\
\hline & $5 \% \mathrm{LC}_{30 \text { min }}$ & $n$ & SSD & $5 \% \mathrm{LC}_{30 \text { min }}$ & $n$ & SSD & $5 \% \mathrm{LC}_{30 \text { min }}$ & $n$ & SSD & $5 \% \mathrm{LC}_{30 \text { min }}$ & $n$ & SSD \\
\hline Standard Probit & $97 \pm 17$ & $3.5 \pm 1.6$ & 325 & $92 \pm 21$ & $1 \pm 0.3$ & 288 & $92 \pm 17$ & $3 \pm 1$ & 241 & $95 \pm 19$ & $1.1 \pm 0.3$ & 256 \\
\hline Loguniform & $78 \pm 16$ & $6 \pm 1.2$ & 459 & $85 \pm 18$ & $1.3 \pm 0.4$ & 413 & $86 \pm 13$ & $4.5 \pm 1.4$ & 247 & $91 \pm 16$ & $1.1 \pm 0.2$ & 241 \\
\hline DEBtox & $69 \pm 54$ & & 5095 & $65 \pm 34$ & & 3721 & $129 \pm 19$ & & 4289 & $107 \pm 14$ & & 1251 \\
\hline \multirow[t]{3}{*}{ Haber TKTD } & $23 \pm 25$ & $1 \pm 0$ & 6451 & $26 \pm 24$ & $1 \pm 0.1$ & 6274 & $31 \pm 20$ & $2 \pm 0.2$ & 6125 & $71 \pm 39$ & $1 \pm 0.1$ & 4814 \\
\hline & \multicolumn{3}{|c|}{ DEBtox slow kinetics } & \multicolumn{3}{|c|}{ DEBtox rapid kinetics } & \multicolumn{3}{|c|}{ Haber TKTD slow kinetics } & \multicolumn{3}{|c|}{ Haber TKTD rapid kinetics } \\
\hline & $5 \% \mathrm{LC}_{30 \text { min }}$ & SSD & & $5 \% \mathrm{LC}_{30 \text { min }}$ & SSD & & $5 \% \mathrm{LC}_{30 \text { min }}$ & $n$ & SSD & $5 \% \mathrm{LC}_{30 \text { min }}$ & $n$ & SSD \\
\hline Standard Probit & $89 \pm 19$ & 391 & & $94 \pm 9$ & 331 & & $109 \pm 21$ & $2 \pm 0.3$ & 507 & $111 \pm 22$ & $2 \pm 0.2$ & 498 \\
\hline Loguniform & $75 \pm 18$ & 515 & & $87 \pm 11$ & 452 & & $91 \pm 26$ & $4.2 \pm 2$ & 888 & $83 \pm 24$ & $4 \pm 2$ & 861 \\
\hline DEBtox & $103 \pm 22$ & 954 & & $101 \pm 7$ & 265 & & $117 \pm 35$ & & 1158 & $117 \pm 35$ & & 1090 \\
\hline Haber TKTD & $15 \pm 3$ & 7024 & & $14 \pm 3$ & 6127 & & $101 \pm 10$ & $2 \pm 0.2$ & 515 & $100 \pm 11$ & $2 \pm 0.2$ & 501 \\
\hline
\end{tabular}


Table 2 Sums of the squares of the deviations between mortality predictions and actual data normalized by dividing by the total number of exposed organisms in the study.

\begin{tabular}{lllll}
\hline & Standard Probit & Loguniform & DEBtox & Haber-TKTD \\
Cyanhydric acid & 3.4 & 3.9 & 2.7 & 12.6 \\
Sulfuric acid & 0.8 & 4.4 & 31.7 & 46.7 \\
Arsine & 2.7 & 5.3 & 3.6 & 5.3 \\
Vinyl chloride & 0.1 & 32.7 & 0.2 & 19 \\
Nitrogen dioxide & 0.5 & 3.3 & 2.2 & 16.7 \\
Sulphur dioxide & 10.9 & 19.6 & 100.8 & 260.6 \\
Hydrogen sulfide & 1.3 & 4.4 & 2.8 & 34.8
\end{tabular}


Table 3 Estimates of $n$ using standard probit or Loguniform models, with for the latter either all the data available or only those for 60 minutes of exposure.

\begin{tabular}{lll}
\hline & Standard Probit & Loguniform with all times \\
Cyanhydric acid & 1.77 & 1.79 \\
Sulfuric acid & 2.92 & 4.42 \\
Arsine & 1.19 & 1.23 \\
Vinyl chloride & 2.38 & 2.41 \\
Nitrogen dioxide & 4.42 & 4.52 \\
Sulphur dioxide & 3.90 & 3.74 \\
Hydrogen sulfide & 2.93 & 2.35
\end{tabular}

